\title{
Metal-free click polymerization of propiolates and azides
}

Researchers from the MOE Key Laboratory of Macromolecular Synthesis and Functionalization, Department of Polymer Science and Engineering, Zhejiang University, China, have expanded the range of monomer pairs used in their established metal-free click polymerization of aroylacetylene-azides to propiolate-azides. They efficiently prepared functional poly(aroxycarbonyltriazole) compounds with aggregation-induced emission characteristics. This study was reported in Volume 54 (Number 4, 2011) of SCIENCE CHINA Chemistry, owing to its significant scientific value.

$\mathrm{Cu}(\mathrm{I})$-mediated alkyne-azide cycloaddition reactions, named "click chemistry" by Sharpless and colleagues, have attracted enormous attention and have been widely used in organic, biological and materials chemistry. Click reactions have also been applied in polymer science, predominantly in the post-functionalization of preformed polymers. While impressive developments have been achieved, fostering the click reaction as a polymerization technique for the synthesis of linear and hyperbranched polymers with advanced functional properties remains challenging. $\mathrm{Cu}(\mathrm{I})$-mediated click polymerization generally suffers from poor solubility, and catalyst residues within polymers can deteriorate their photophysical properties.

Professor Tang's research group has developed the syntheses of polytriazoles by click polymerization, by extending their research expertise in triple-bond chemistry. They have prepared functional linear and hyperbranched polytriazole compounds from organo-soluble $\mathrm{Cu}\left(\mathrm{PPh}_{3}\right)_{3} \mathrm{Br}$-catalyzed click polymerization. To solve the problem of catalyst residues in polymers, they have established an efficient metal-free click polymerization of aroylacetylenes and azides, and synthesized readily soluble high molecular weight poly(aroyltriazole) compounds in high yield and short reaction times. The preparation of aroylacetylenes still requires many reaction steps and harsh reaction conditions.

In this work, Qin, Tang and coworkers extended the range of monomer pairs used in the metal-free click polymerization of aroylacetylene-azides to propiolate-azides, according to structural similarities of the alkynes. Propiolates were easily prepared from commercially available propiolic acid and diols via a single-step one-pot esterification under moderate reaction conditions. The metal-free click polymerization of 4,4'-isopropylidenediphenyl dipropiolate and tetraphenylethene (TPE)-containing diazides was carried out in a polar solvent at moderate temperatures for a short time. The reaction produced poly(aroxycarbonyltriazole) compounds (PACTs) with high molecular weight (weight-average molecular weight up to 23900) and regioselectivity (fraction of 1,4-isomers up to 90\%) in high yield (up to 99\%). Polymers exhibited unique aggregation-induced emission characteristics owing to TPE units, and their nanoaggregates functioned as sensitive fluorescent chemosensors for the superamplified detection of explosives. Since no metallic catalyst was used, such polymerization processes are less toxic and more environmentally. The metal-free click polymerization of propiolates and azides paves the way for the generation of PACTs with new topological structures and advanced functional properties.

One reviewer is quoted as saying "This paper is an extension of the authors' earlier work $\cdots$ The work is very interesting and of academic significance." The study of the facile synthesis of poly(aroxycarbonyltriazole) compounds with aggregation-induced emission characteristics by metal-free click polymerization by Qin et al. has been published in Volume 54 (Number 4, 2011) of SCIENCE CHINA Chemistry. "This paper enriches and further develops the 'Metal-free Click Polymerization' established by the authors," said another reviewer.

The authors' group is predominantly focused on developing polymerization methods based on triple-bond building blocks, the synthesis of functional polymeric materials, and the exploration of new molecular systems with aggregation-induced emission characteristics and appropriate applications.

This work was supported by the National Natural Science Foundation of China (20634020, 50703033, 20974098 and 20974028), the Ministry of Science and Technology of China (2009CB623605), the University Grants Committee of Hong Kong (AoE/P-03/08 and HKUST2/CRF/10), the Research Grants Council of Hong Kong (603509 and 601608), the Innovation and Technology Fund of Hong Kong (ITS/168/09), the Postdoctoral Research Foundation of China (2008461), and the Fundamental Research Funds for the Central University (2010KYJD005).

See the article: Qin A, Lam J W Y, Tang B Z. Click Polymerization. Chem Soc Rev, 2010, 39: 2522-2544

Qin A, Lam J W Y, Tang B Z. Click Polymerization: Progressed horoscope, challenges, and opportunities. Macromolecules, 2010, 43: 8693-8702

Li H, Mei J, Wang J, et al. Facile synthesis of poly(aroxycarbonyltriazole)s with aggregation-induced emission characteristics by metal-free click polymerization. Sci China Chem, 2011, 54: 611-616

Open Access This article is distributed under the terms of the Creative Commons Attribution License which permits any use, distribution, and reproduction in any medium, provided the original author(s) and source are credited. 